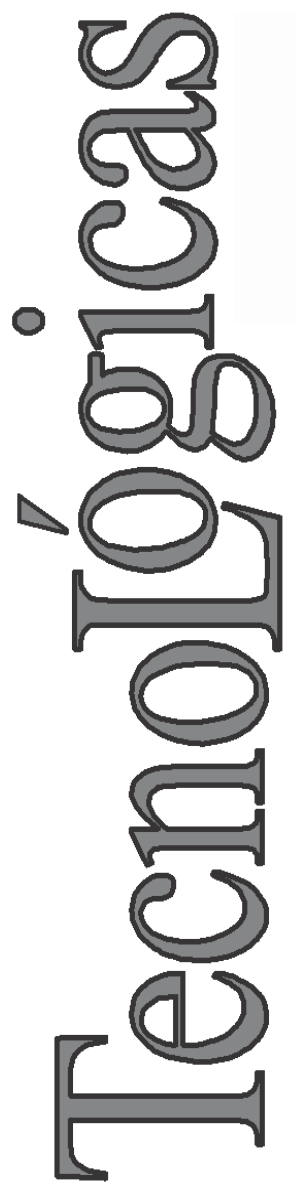

\title{
Diseño de Producto Basado en la Matriz de Funciones y Componentes
}

\section{Product Design Based on Matrix Functions and Components}

\author{
Oscar E. Herrera-Bedoya ${ }^{1}$
}

Daniel A. Bojacá ${ }^{2}$

1 Programa Ingeniería de Sistemas, Universidad Piloto de Colombia, Bogotá-Colombia oscar-herrera@unipiloto.edu.co,

2 Programa Ingeniería de Sistemas, Universidad Piloto de Colombia, Bogotá-Colombia daniel-bojaca@unipiloto.edu.co 


\section{Resumen}

Se presenta una herramienta para el diseño de producto basada en la reordenación de la matriz de funciones y componentes. Esta herramienta ha sido desarrollada en MATLAB y utiliza un primer proceso basado en la diagonalización, un segundo proceso basado en el intercambio de elementos y finalizado con una inspección visual. Los resultados obtenidos fueron la reducción de carga computacional y una aproximación entre el $90 \%$ al 95\% del mínimo global de la entropía. Esto permite que un proceso de toma de decisiones sea más veloz manteniendo un gran porcentaje de exactitud y/o organizarlos para facilitar su manipulación.

\section{Palabras clave}

Selección de procesos; entropía; ordenación de matrices; optimización.

\section{Abstract}

It's presented a tool for product design based on the rearrangement of features and components matrix. This tool has been developed in MATLAB and uses a first process based on the diagonalization, a second process based on the exchange of items and ends with a visual inspection. The results obtained were the reduction of computational cost and an approximation between $90 \%$ to $95 \%$ of the global minimum of entropy. This allows a faster decision-making process with a high percentage of accuracy and/or organize for easier handling.

\section{Keywords}

Decision-making process; entropy; matrix rearrangement; optimization. 


\section{INTRODUCCIÓN}

Todos albergamos en nuestra mente una idea intuitiva del desorden. Sin embargo, hay que tener claro que en términos científicos, el desorden viene dado por el número de estados en los que un sistema puede estar. Para medir el orden o desorden se usa la entropía, la cual no está definida como una cantidad absoluta (Ет), sino como la diferencia entre la entropía inicial de un sistema $\left(\mathrm{E}_{\mathrm{I}}\right)$ y la entropía final del mismo $\left(\mathrm{E}_{\mathrm{F}}\right)$. Solo tiene sentido hablar de entropía en términos de un cambio en las condiciones de un sistema.

Hoy en día, existen herramientas para calcular la mínima entropía en los procesos del diseño de productos y se han desarrollado diferentes métodos de selección de diseño, basados en criterios de desempeño o en conceptos funcionales dejando a un lado métodos y criterios de selección de los procesos en una línea de producción. Los procesos de producción, constituyen un eslabón clave de la organización para responder de manera efectiva y distintiva al cúmulo creciente de necesidades de los clientes, para lo cual es necesario diseñar, formular y poner en práctica estrategias de producción adecuadas y eficientes. Además, la calidad de un producto es una resultante, que emerge debido a una interrelación de un conjunto de procesos que tienen lugar dentro de las organizaciones. Muchas veces se ignoran o subvaloran las características tan complejas que poseen tales procesos, incluso algunas personas y empresas consideran que el proceso de producción es el único responsable de lograr la calidad que los clientes esperan y exigen.

Así pues, este artículo muestra el desarrollo de una herramienta basada en ordenaciones matriciales, para minimizar la entropía existente entre los diferentes procesos de la línea de producción/manufactura de un producto en una empresa. Esta herramienta minimiza en un alto porcentaje la entropía, de forma más rápida y versátil, además, el aspecto visual de esta matriz es más amigable e intuitivo, utilizándose un proceso matemático directo y una reordenación iterativa que evalúa un conjunto de posibles soluciones, y escogiendo de estas el mejor resultado, se tiene entonces un método mixto que utiliza un cierto porcentaje de fuerza bruta con una estrategia predefinida. 


\section{DESARROLLO}

Para facilitar la toma de decisiones en el diseño o procesos de productos, se construye una matriz de funciones-subsistemas denominada matriz A (Tabla 1). En esta matriz se identifican las funciones que debe cumplir el sistema (producto) colocándolas en las filas, luego se determinan los pesos de cada una utilizando la matriz de dominación, y también se identifican los componentes del sistema que pueden cumplir dichas funciones y sus pesos ubicándolos en las columnas. Se debe tener en cuenta que la sumatoria de los valores de cada fila deben estar normalizados, de esta manera, el valor de cada elemento de la matriz representa el grado de cumplimiento de la función por parte del componente correspondiente.

Tabla 1. Formato Matriz A

\begin{tabular}{|l|c|c|c|}
\hline \multicolumn{3}{|c|}{ Componentes } \\
\hline \multirow{3}{*}{ Funciones } & & $C i$ & $E_{f}$ \\
\cline { 2 - 4 } & $W j$ & Matriz & \\
\cline { 2 - 4 } & & $E_{c}$ & \\
\hline
\end{tabular}

Por ejemplo en el diseño de un carro o en la línea de producción, las funciones pueden ser la ergonomía, la seguridad o la estética y los componentes, la silla, el volante y la carrocería, véase la Tabla 2.

Tabla 2. Ejemplo Matriz A

\begin{tabular}{|l|c|c|c|c|c|}
\cline { 3 - 6 } \multicolumn{2}{c|}{} & Silla & Volante & Carrocería & \\
\cline { 3 - 6 } \multicolumn{2}{c|}{} & 0,25 & 0,50 & 0,25 & 1 \\
\hline Ergonomía & 0,4 & 0,40 & 0,60 & & 1 \\
\hline Seguridad & 0,4 & & 0,20 & 0,80 & 1 \\
\hline Estética & 0,2 & 0,75 & & 0,25 & 1 \\
\hline & 1 & & & & \\
\hline
\end{tabular}

Con el fin que esta matriz colabore con el proceso de diseño, interesa "reordenar" la matriz A, de modo que se puedan identificar de forma visual tanto grupos de elementos que tengan en común el cumplimiento de una misma función, como grupos de funciones que tengan en común el uso del mismo componente. De esta mane- 
ra, la matriz A tendrá elementos agrupados, permitiendo identificar submatrices que facilitan la toma de decisiones. Para esto es importante el concepto de entropía, Yeung (2002), que se define como la dispersión entre elementos de una misma fila, de modo que el valor de la entropía es menor, si los elementos con un valor diferente de 0 están agrupados.

Para el cálculo de la menor entropía total y la reordenación de la matriz A, se realizan dos procesos, el primero basado en el movimiento de las columnas y el segundo en el movimiento de las filas. La entropía inicial total $E_{T}$ del sistema $(A)$, viene dada por ¡Error! No se encuentra el origen de la referencia..

$E_{T}=E_{F}+E_{C}$

La entropía funcional está dada por (2):

$E_{F}=\sum_{i=1}^{|f|}\left(w_{i} \sum_{j=1}^{|C|}\left(v_{i j}\left|d_{i j}\right|\right)\right) ; \quad d_{i j}=y_{i}-x_{j}, v_{i j} \neq 0$

Donde $|\mathrm{f}|$ es el número de funciones y $|\mathrm{C}|$ el número de componentes, $i, j$ es el número de la función (fila) y del componente (columna) respectivamente. $S_{i j}$ es la componente que viene descrita por su posición $x_{i j}$ y su peso $v_{i j}, x_{j}$ es la posición $j$ del elemento $S_{i j}$ respecto a cada fila $i, v_{i j}$ es el peso del componente $S_{i j}$, sumatoria para cada valor de fila $i$ debe ser igual a uno, $y_{i}$ es el valor de $x_{i j}$ con mayor peso $v_{i j}$ asociado respecto a cada fila $i, d_{i j}$ es la distancia del componente analizado $S_{i j}$ respecto al componente más influyente $y_{i}$ de cada fila $i$ dada, y $W_{i}$ es el peso de la función en el sistema. La entropía por componentes está dada por (3):

$E_{C}=\sum_{j=1}^{|C|}\left(C_{j} \sum_{x=1}^{|f|}\left(v_{i j}\left|d_{i j}\right|\right)\right) ; d_{i j}=y_{j}-x_{i}, v_{i j} \neq 0$

Donde $x_{i}$ es la posición $i$ del elemento $S_{i j}$ respecto a cada columna $j, y_{j}$ es el valor de $x_{i j}$ con mayor peso $v_{i j}$ asociado respecto a cada columna $j, d_{i j}$ es la distancia del componente analizado $S_{i j}$ 
respecto al componente más influyente $y_{j}$ de cada columna $j$ dada, y $C_{j}$ es el peso general del componente en el sistema.

En González (2001), la ordenación del sistema es realizada en dos procesos, el primer proceso se realiza con el algoritmo llamado de reordenación de la matriz A por entropía funcional, que agrupa los componentes que cumplen una determinada función, el cálculo de la entropía en la primera función $E_{F}$ está dado por (2).

En este algoritmo, primero se identifica el componente de mayor valor, es decir, el que da mayor cumplimiento a la función y por lo tanto más influyente $y_{i}$, luego se analiza la posición del elemento $S_{i j}$ que corresponda al componente que le sigue en importancia en cuanto al cumplimiento de la función, colocándolo en la columna más cercana posible al mayor valor (intercambio de columnas). Al realizar este intercambio se calcula el nuevo valor de $E_{T}$ y si es menor que el anterior entonces se consolida el cambio. Se repite proceso, eligiendo la siguiente función en importancia (peso).

El segundo proceso en González (2001), se realiza mediante el algoritmo llamado de reordenación de la Matriz A por entropía de componente, que agrupa las funciones que cumple un determinado componente. Se realiza análogamente al primero, de este modo el primer paso es hallar el componente de más peso y optimizar respecto a su entropía por medio del intercambio de filas. Éste método iterativo converge rápidamente a un mínimo que no siempre obedecerá a un mínimo global, ya que no evalúa paralelamente un conjunto de soluciones, si no que secuencialmente y va fijando posiciones al alcanzar lo que a priori se considera un mínimo.

\section{METOdOLOGía}

Para el desarrollo de la herramienta, se ha tomado como punto de partida la matriz A, con los pesos de las filas (funciones) y de las columnas (componentes) ya asignados. Primero se tratará la reordenación de funciones y de la misma forma se realizará la ordenación por componentes. Se tiene entonces, que el conjunto de soluciones $\mathrm{X}$ debe ser un conjunto de números enteros positivos, que representa las posiciones de los elementos en las $n$ posibles 
posiciones que deben ocupar los componentes y las funciones en la matriz A para minimizar la entropía total del sistema.

$X=\left[X_{1}, \ldots, X_{|C|}\right]$

Existen por tanto restricciones; y una solución es factible si se verifica que en el vector $\mathrm{X}_{i}$ no existen valores $x$ repetidos

$x_{k} \neq x_{p} \Rightarrow k \neq p$

Una solución se considera óptima si es factible y minimiza la función objetivo que viene dada por (6):

$f\left(x_{i}\right)=E_{F}$

Se tiene un problema de optimización donde hay que determinar los valores de $x_{i}$ que minimicen el valor de $f\left(x_{i}\right)$

$$
\min _{x_{i} \in \mathbb{Z}^{+} \mid x_{k} \neq x_{p} \Rightarrow k \neq p} f\left(x_{i}\right)
$$

Análogamente en la ordenación por componentes se tiene que la función objetivo está dada por

$f\left(x_{j}\right)=E_{C}$

Siendo para éste caso el problema de optimización:

$\min _{x_{j} \in \mathbb{Z}^{+} \mid x_{k} \neq x_{p} \Rightarrow k \neq p} f\left(x_{j}\right)$

La solución más obvia es evaluar la solución objetivo con todas las posibles combinaciones es decir $|C| !+|f|$ ! que crece factorialmente con el número de componentes y funciones que intervienen en el sistema (Frederick \& Lieberman, 2002). Para solucionar este problema, se ha implementado una herramienta por medio de $M A T L A B$, con la cual se logró mejorar el tiempo y la cantidad de iteraciones para calcular una entropía entre el $90 \%$ - 95\% del mínimo global. 
Esta aplicación permite una gran exactitud que, a posteriori mediante una simple inspección visual logra alcanzar el mínimo valor de la entropía.

Partiendo de la matriz A, el primer paso es hacer en MATLAB, una diagonalización utilizando el método de "Column approximate minimum degree permutation" (Larimode et al., 2006), el cual busca diagonalizar todos los elementos diferentes de 0 de una matriz dispersa, ésta diagonalización no tiene en cuenta el peso de los elementos que componen la matriz, pero permite obtener un punto de partida con una tendencia de la diagonal.

Luego, se ejecuta el método que se ha denominado en este trabajo permutación deslizante. Este método toma el grupo de elementos, los permuta y desliza, obteniendo una matriz de posibles soluciones, calculando al final la $E_{T}$ de cada posible solución y escogiendo el orden que menor resultado produzca. En la permutación deslizante lo que se hace es obtener la matriz de permutaciones de las posiciones donde $v$ es distinto de cero, para una $i$ o $j$ dada dependiendo del tipo de ordenación (funcional o por componentes). Por ejemplo, teniendo:

$x=[1 \cdots n]$

Y siendo el conjunto de vectores $r$ los valores de $\mathrm{x}$ donde $v_{i j}$ son distintos de cero.

$r_{1}=\left[\begin{array}{llll}2 & 5 & \cdots & \left.n_{1}\right|_{v_{1,|C|} \neq 0}\end{array}\right]$

$r_{|f|}=\left[\begin{array}{llll}2 & 3 & \cdots & \left.n_{1}\right|_{v_{|f|,|,|} \neq 0}\end{array}\right]$

A éste vector $r$ lo permutamos obteniendo una matriz que deslizamos a través del vector $x$, obteniendo la matriz de permutaciones $\bar{X}$.

$\bar{X}=\left[\begin{array}{ccccc}{\left[\operatorname{perm}\left(r_{j}\right)\right]} & 1 & \cdots & |C|-1 & |C| \\ 1 & {\left[\operatorname{perm}\left(r_{j}\right)\right]} & \ldots & |C|-1 & |C| \\ 1 & \cdots & |C|-1 & {\left[\operatorname{perm}\left(r_{j}\right)\right]} & |C| \\ 1 & \cdots & |C|-1 & |C| & {\left[\operatorname{perm}\left(r_{j}\right)\right]}\end{array}\right]$ 
La cantidad de operaciones que se realizan en la permutación deslizante se puede obtener por medio de (13).

$O p=\sum_{i=1}^{|f|}\left(|C|-n_{i}+1\right) n_{i} !+\sum_{j=1}^{|C|}\left(|f|-n_{j}+1\right) n_{j} !$

Siendo el número de elementos $n$, los elementos distintos a cero de cada función $\left(n_{i}\right)$ o los elementos distintos a cero que siendo del mismo componente intervienen en distintas funciones $\left(n_{j}\right)$. $\mathrm{Al}$ evaluar el conjunto de soluciones $\bar{X}$ en las ecuaciones de entropía se escogerá el que menor resultado obtenga, y determinando de ésta manera el vector de soluciones óptimo.

\section{RESULTADOS}

El punto de partida de la aplicación es la matriz A de la Fig. 1. Esta matriz relaciona las funciones de un producto (filas) con los componentes (columnas), que realizan determinada función. La idea principal es "reordenar" la matriz para obtener la menor entropía ¡Error! No se encuentra el origen de la referencia. que ayude a una rápida inspección visual. En la Fig. 1, se muestra el formato en el que se representan las matrices. Siendo C el vector peso de los componentes, $\mathrm{W}$ el peso de cada función y $E_{C}$ y $E_{f}$ las entropías de los componentes y funcional respectivamente.

En la Fig. 1, se identifica y representa mediante un círculo el componente con el máximo valor en cada función y con una $\mathbf{X}$ la función con máximo valor en cada componente. Se calcula $E_{T}$ inicial de la matriz A, utilizando (1) y el valor obtenido es 2,06 . Si se quisiera encontrar el orden en el que deberían ir las columnas y filas (X) lo que primero se pensaría es en encontrar todas las posibles combinaciones de ordenación que nos llevara a la menor entropía. Entonces sería necesario evaluar 41040 combinaciones para encontrar por fuerza bruta la menor entropía global del sistema. Para al final encontrar 8 posibles ordenaciones (señaladas con círculo rojo) que nos darían el mínimo global de la entropía. 


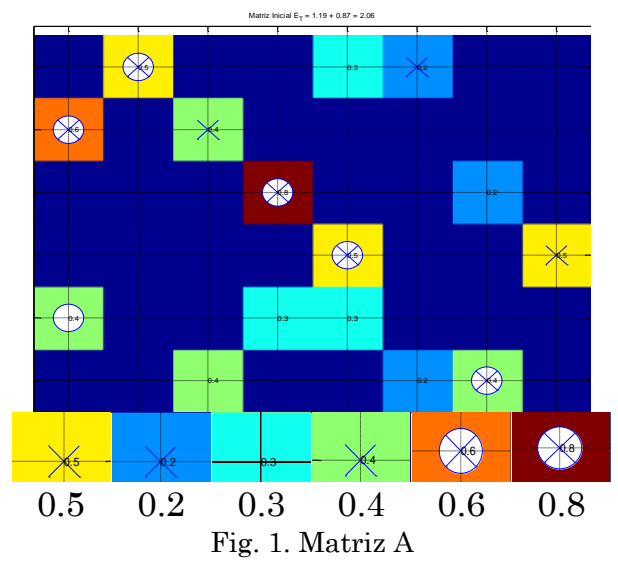

Lo que se plantea es reducir ese número alto de permutaciones, utilizando inicialmente un método matemático y posteriormente uno iterativo que se ha denominado permutación deslizante. Para obtener una primera reordenación de la Matriz A, se aplica la función de MATLAB "Column approximate minimum degree permutation" (Fig. 2a), como resultado se tiene una minimización de $E_{T}$ y una agrupación inicial de elementos. Después de aplicar esta función la entropía se ha reducido a 1,609 de los 2,06 iniciales. Esto permite obtener una ordenación base tanto de filas como de columnas para el siguiente paso. Es decir, las filas estarán ordenadas 4, 5, 1, 6, 3 y 2 y las columnas $3,1,5,4,7,6,2$ y 8 . A continuación, el proceso iterativo trata de deslizar la matriz de permutación partiendo de la ordenación de la diagonalización.

$P=\left[\begin{array}{cccccccc}5 & 8 & 3 & 1 & 4 & 7 & 6 & 2 \\ 8 & 5 & 3 & 1 & 4 & 7 & 6 & 2 \\ 3 & 5 & 8 & 1 & 4 & 7 & 6 & 2 \\ 3 & 8 & 5 & 1 & 4 & 7 & 6 & 2 \\ \vdots & \vdots & \vdots & \vdots & \vdots & \vdots & \vdots & \vdots \\ 5 & 4 & 7 & 6 & 2 & 8 & 1 & 3 \\ 5 & 4 & 7 & 6 & 2 & 8 & 3 & 1\end{array}\right]$

Al calcular todas las entropías del conjunto de soluciones $P$, y al identificar la entropía mínima, se obtiene el resultado mostrado 
en la Fig. 2b. Luego el proceso se repite para la ordenación por componentes y se obtiene la matriz de la Fig. 3a.

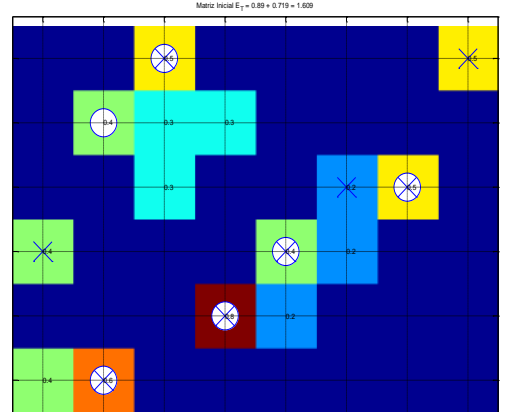

a)

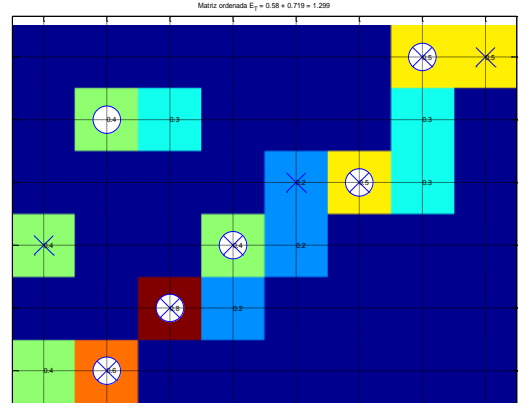

b)

Fig. 2. Matriz reordenada, a) Matriz A después de la primera "reordenación", b) Matriz con ordenación funcional

Con este método, se ha alcanzado aproximadamente un 95\% del mínimo global $(1,005)$. Y finalmente con una inspección visual intercambiando la fila 4 y 1 y reordenando las cuatro primeras columnas a 7431 , se obtiene una entropía en un mínimo global de 0,953 (Fig. 3b). Se puede observar que la matriz de la Fig. 3b, aunque tiene una entropía menor $(0,953)$ es estéticamente menos atractiva que la matriz del sistema de la Fig. 2 b con una entropía de 1,005 que claramente muestra una tendencia diagonal y subsistemas agrupados.

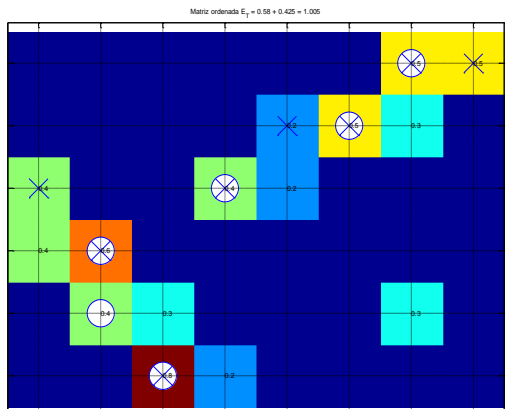

a)

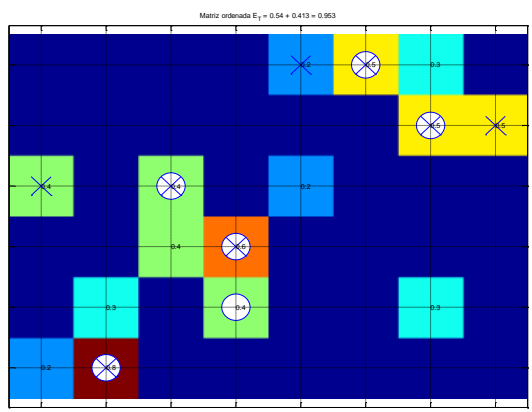

b)

Fig. 3. Matriz Final, a) Matriz con ordenación por componentes partiendo de la Ordenación Funcional, b) Matriz después de la inspección visual 


\section{CONCLUSIONES}

La importancia de éste método está en la ayuda visual que proporciona, facilitando enormemente la toma de decisiones de carácter intuitivo a miembros de equipos que están en el campo. En un método completo, se tendrían que realizar $M !+N$ ! permutaciones (fuerza bruta), para alcanzar la reordenación óptima que garantice un mínimo global en la denominada entropía total del sistema, este gran número de permutaciones puede tener un costo computacional de gran importancia que restaría funcionalidad a la reordenación. Sin embargo, con el método presentado se logra obtener una cercanía al mínimo global del $95 \%$, con un número de operaciones iterativas proporcional al número de componentes que interfieren en una función y no al número de componentes existentes en el sistema, y del mismo modo con las funciones.

Como trabajo futuro se está trabajando actualmente en incluir en el método una optimización meta-heurística que permita encontrar mínimos locales con un menor número de iteraciones y para tamaños de matrices aún mayores, para ello se está utilizando Optimización con enjambre de partículas (Particle Swarm Optimization - $P S O$ ) que es un algoritmo metaheurístico evolutivo inspirado en el comportamiento social de las bandadas de pájaros o bancos de peces desarrollada por J. Kennedy y R. C. Eberhart en 1995. Este método es un algoritmo iterativo cuya base es una población de individuos denominada enjambre, en la que cada individuo, llamado partícula, explora el espacio de decisión en busca de las soluciones óptimas. Cada partícula evalúa su posición relativa al objetivo (fitness) en cada iteración, y las partículas en el entorno comparten los recuerdos de sus "mejores" posiciones para ajustar sus velocidades y siguientes posiciones con objeto de obtener en un futuro el objetivo buscado.

\section{REFERENCIAS}

Frederick, H, Gerald, L., (2002). Investigación de Operaciones. McGrawHill.

González, M.C., (2001). Implantación Y Aplicación De Una Estrategia General, Basada En La Teoría De Las Dimensiones Del Proyecto, Pa- 
ra La Elaboración De Una Metodología De Resolución De Layout. Estructuración De Las Técnicas Necesarias. Tesis Doctoral (Dir. GómezSenent Martínez, Eliseo)

Horn, R.A., Johnson, C.R., (1990). Matrix Analysis. Cambridge University Press.

Kennedy, J., Eberhart, R.C., (1995). Particle swarm optimization, in Proc. IEEE Conf. Neural Networks IV, Piscataway, NJ.

Larimore, S.I., Davis, T.A., Gilbert, J., \& Esmond, N., (2006). Column approximate minimum degree permutation Sparse Matrix Algorithms Research at the University of Florida: http://www.cise.ufl.edu/research/sparse/

Yeung, W., (2008). A first course in Information Theory. Springer. 